\title{
Linking Necessity to Apriority
}

Final Draft - Published in Acta Analytica (2018)

\author{
Tristan Haze \\ The University of Sydney \\ tristanhaze@gmail.com
}

\begin{abstract}
There is an important and fairly straightforward link between necessity and apriority which can shed light on our knowledge of the former, but initially plausible attempts to spell out what it is fall victim to counterexamples. Casullo (2003) discusses one such proposal, argues - following Anderson (1993) - that it fails, and suggests an alternative. In this paper, I argue that Casullo's alternative also fails, before making a suggestion for which I can find no counterexamples and which, notably, handles some recent examples due to Kipper and Strohminger \& Yli-Vakkuri.
\end{abstract}

Keywords: necessity, apriority, epistemology.

\section{The task: extending Kripke's 'philosophical analysis' point}

The object of this paper is to establish a certain kind of link between (metaphysical, subjunctive) necessity and apriority, a link which shows a way in which our knowledge of necessity can always be based on an a priori factor. This description of what is shown by the link I seek to establish is of course not very precise. I propose to explain more clearly what I have in mind by, firstly, differentiating the task attempted by the present paper from two distinct and more ambitious tasks attempted in the philosophical literature, and secondly, by drawing attention to a link between certain examples of necessities and apriority which we find articulated in Kripke (1980). It is the present paper's task to extend this link to all propositions which can be known to be necessary (in the sense of 'non-contingent', i.e. 'necessarily true or necessarily false', which is the sense in which I will use 'necessary' throughout this paper).

The aforementioned two more ambitious tasks which are also about linking necessity and apriority are those of epistemic two-dimensionalism ${ }^{1}$ and principles-based approaches to modal knowledge ${ }^{2}$. Roughly speaking, the epistemic two-dimensionalist seeks to construct or characterize maximally specific scenarios using language that doesn't lead to necessary a posteriori propositions, and then to give an analysis on which the truth-value of necessity-claims can depend on the a posteriori matter of which scenario is actual. This counts as an attempt to forge a link between necessity and apriority, since the characterization of the underlying space of scenarios is given in terms of what an ideal reasoner could not rule out. The principles theorist seeks to specify general a priori principles which, together with empirical input in at least some cases, yield as a

\footnotetext{
${ }^{1}$ E.g. Chalmers (2006, 2006b, 2011).

${ }^{2}$ E.g. Peacocke (1998). For ease of exposition, the principle I use as an example - Necessity of Identity - is just a familiar Kripkean doctrine. Peacocke's account attempts to go deeper, with a principle governing how extensions are assigned to terms. This difference does not matter for the purposes of the contrast being drawn here.
} 
consequence the result that particular propositions we may be interested in are necessarily true. For example, our knowledge of the a priori principle of the Necessity of Identity and our empirical knowledge that Hesperus is Phosphorus go together to give us the knowledge that 'Hesperus is Phosphorus' is necessarily true. The principles theorist attempts to link necessity to apriority by linking our knowledge that certain propositions are necessary back to our knowledge of certain general a priori principles.

By contrast, the present paper seeks to link the knowledge of the necessity of propositions back to a priori knowledge about those necessary propositions themselves (or, to anticipate a bit, propositions which imply them or their negations), and these necessary propositions may be quite specific and non-principle-like. (To be sure, there may in turn be general principles underlying this a priori knowledge, but that is another matter which we don't need to settle in order to forge the link we are after here.) While perhaps falling short of having the full significance that epistemic two-dimensionalism or principles-based approaches may be expected to have, this link is instructive, well worth articulating, and fairly straightforward. Furthermore, as we shall see, this task has already been attempted in the literature, but without full success.

To get a more concrete idea of the task, consider canonical Kripkean examples of the necessary a posteriori: 'Cats are animals', 'Water is $\mathrm{H}_{2} \mathrm{O}$ ', and 'Hesperus is Phosphorus'. While these propositions cannot be known a priori, it can be known a priori - by 'a priori philosophical analysis', as Kripke puts it ${ }^{4}$ - that they are necessarily true if true at all. The task is simply to extend this insight so that it covers all necessary propositions, or at least all propositions which can be known to be necessary. In the next section, I will - following Casullo (2003), who followed Anderson (1993) - consider the most obvious extension of this insight and why it fails. In section 3, I will consider a more sophisticated extension due to Casullo, and argue that it fails too. In section 4 I will explain my proposed extension.

Before continuing, a word on the starting assumptions of this paper. I am assuming that sense can be made of the Kripkean notion of necessity, and that there are cases of the necessary a posteriori. I am assuming that sense can be made of the notion of knowability a priori, roughly along traditional lines: knowability without recourse (in a certain sense) to experience (in a certain sense). Finally, my proposal helps itself to talk of implication or logical consequence construed as a relation between (sets of) propositions. If you favour the view that logical consequence is a relation between (sets of) sentences, or if you have a coarse-grained notion of propositions for which there may be no available notion of implication suitable for my purposes, I invite you to read 'proposition' throughout this paper as 'sentence' or 'statement'. ${ }^{5}$ While I do not specify a particular notion of implication, I am not thereby committing myself to logical monism; I just need there to be at least one near-to-hand relation which behaves in the way I require. If monism is the case, then my talk of implication is unambiguous. If pluralism is the case, it is perhaps ambiguous, and there may be further work to do investigating how the different disambiguations fare.

\section{The obvious extension and why it fails}

\footnotetext{
${ }^{3}$ I will not be worrying here about whether these examples are genuine. If you believe in the necessary a posteriori for broadly Kripkean reasons but prefer other examples, you should be able to adapt what I have to say to them without much trouble.

${ }^{4}$ Kripke (1971, p. 153.)

${ }^{5}$ Thanks to an anonymous referee for highlighting this point.
} 
Kripke made the point that, while his examples of the necessary a posteriori are indeed a posteriori, they can be known a priori to be necessary (in the sense of 'either necessarily true or necessarily false' which I will stick to in this paper). The most obvious way of extending this point is simply to propose that what Kripke pointed out about his examples is true of all necessary propositions (and perhaps that the same holds for contingent propositions). In Casullo's (2003) terminology: while Kripke showed that knowledge of specific modal status (necessarily true, contingently false, etc.) is not always possible a priori, this leaves open that general modal status (necessary or contingent, with truth value left open) may be. Briefly put (for the case of necessity):

(Obv1): All necessary propositions can be known a priori to be necessary.

You might worry about propositions whose general modal status cannot be known at all, for instance because those propositions aren't entertainable. To get around this worry, let us following Casullo (2003) - conditionalize as follows:

(Obv2) If $p$ is a necessary proposition and $\mathrm{S}$ knows that $p$ is a necessary proposition, then $\mathrm{S}$ can know a priori that $p$ is a necessary proposition.

Casullo, following Anderson (1993), argues convincingly that (Obv2) is false. Consider:

(ObvX) Hesperus is Phosphorus or my cup is on the table.

This is a necessary proposition, but for all anyone could know a priori, it could be necessarily true (if the first disjunct is true), contingently true (if the first disjunct is false but the second true), or contingently false (if both disjuncts are false). So (Obv2) can't be right.

\section{Casullo's extension and why it fails}

In an interesting effort to avoid the problem affecting (Obv2), Casullo introduces the following notions of conditional modal propositions and conditional modal status:

Associated with each truth functionally simple proposition is a pair of conditional propositions: one provides the specific modal status of the proposition given that it true; the other provides its specific modal status given that it is false. Associated with each truth functionally compound proposition is a series of conditional propositions, one for each assignment of truth values to its simple components. Each conditional proposition provides the specific modal status of the proposition given that assignment of truth values. Let us call these propositions conditional modal propositions and say that $\mathrm{S}$ knows the conditional modal status of $p$ just in case $\mathrm{S}$ knows all the conditional modal propositions associated with $p$. (Casullo (2003), p. 197.)

His proposed link between necessity and apriority is as follows:

(Cas) If $p$ is a necessary proposition and $S$ knows the conditional modal status of $p$, then $S$ can know a priori the conditional modal status of $p$.

Casullo dubs this 'a version of the traditional account of the relationship between the a priori and the necessary that is immune to Kripke's examples of necessary a posteriori 
propositions' (Casullo (2003), p. 199). (Cas) handles (ObvX) nicely. Calling (ObvX)'s disjuncts 'Hesp' and 'Cup', its associated conditional modal propositions will run as follows:

If Hesp is true and Cup is true, (ObvX) is necessarily true.

If Hesp is true and Cup is false, (ObvX) is necessarily true.

If Hesp is false and Cup is true, (ObvX) is contingently true.

If Hesp is false and Cup is false, (ObvX) is contingently false.

These are plausibly knowable a priori, as required by (Cas).

But consider:

(CasX) Everything is either such that it is either not Hesperus or is Phosphorus, or such that it is either on the table or not my cup.

While it contains connectives, this is not a truth functional compound in the relevant sense, since it does not embed any whole propositions. So on Casullo's proposal, (CasX) will be associated with just a pair of conditional modal propositions. Which ones? A problem here is that there is no very clear positive case for any pair (the proposal, after all, was not formulated with (CasX) in mind), but I think it is clear that the only candidate pair which could stand a chance is:

If (CasX) is true, it is necessarily true.

If (CasX) is false, it is contingently false.

(After all, (CasX) is true and necessary, so the other available choice for first member couldn't be right, and the second member of the pair seems true and knowable a priori.)

Instantiating Casullo's proposal (Cas) on (CasX), we get:

If (CasX) is a necessary proposition and $\mathrm{S}$ knows the conditional modal status of (CasX), then $\mathrm{S}$ can know a priori the conditional modal status of (CasX).

But it is clear that the first conditional modal proposition for (CasX), i.e. that if (CasX) is true, it is necessarily true, could not be known a priori. So (Cas) can't be right either. ${ }^{6}$

\section{A better extension: bring in implication}

Something which strikes one initially about (ObvX), the counterexample to (Obv2), is that it has a component which can be known a priori to be necessarily true if true at all. But this isn't true of (CasX); it has no component propositions at all. What I suggest is true and important about both counterexamples is, not that they have component propositions which can be known a priori to be necessarily true if true at all, but that they are implied by such propositions.

\footnotetext{
${ }^{6}$ You may have noticed that, if we put ourselves in the $S$ role, there is something funny about saying we know the first conditional modal proposition, since we know the consequent already. But this can be gotten around by imagining that we don't know whether Hesperus is Phosphorus, and are just told by somebody trustworthy: 'Look, I'm not going to tell you whether Hesperus is Phosphorus or not, but I will tell you that (CasX) is either false or necessarily true'. Then, on the basis of that knowledge, we could infer the first conditional modal proposition. But we could not have known it a priori.
} 
Let us say that a proposition $p$ possesses the Kripkean property iff it can be known a priori that it is necessarily true if true at all. Now, I submit that if a proposition whose general modal status is knowable at all is necessarily true, then it is in the deductive closure of a set of true propositions possessing the Kripkean property. More generally, if a proposition whose general modal status is knowable at all is necessary, then either it or its negation is in the deductive closure of a set of true propositions possessing the Kripkean property. To cast the point in a form similar to (Obv2) and (Cas) above:

(Link) If $p$ is a necessary proposition and $\mathrm{S}$ knows that $p$ is a necessary proposition, then either $p$ or its negation is in the deductive closure of a set of true propositions which $S$ can know a priori to be necessarily true if true at all.

I have not been able to find any counterexamples to (Link). Furthermore, in contrast to principles recently suggested in Casullo (forthcoming) and Kipper (2018), and criticised in Strohminger \& Yli-Vakkuri (m.s.), (Link) is not restricted to atomic propositions.

In closing, I note that (Link) can handle two recently discussed examples, due to Kipper $(2018, \S 3)$ and Strohminger \& Yli-Vakkuri (m.s., p. 7), which make their own trouble for Casullo's proposal.

Kipper's example is 'Air is airy stuff'. (Kipper uses 'airy stuff' as a shorthand for the superficial characteristics that air has in our world.) It turns out that all there is to being air is being airy stuff, but since it cannot be known a priori that air is not a natural kind, 'Air is airy stuff' does not possess the Kripkean property. Our knowledge that it is necessary stems from our empirical knowledge that air is not a natural kind.

Strohminger \& Yli-Vakkuri's example is 'Eminem is not taller than Marshall Mathers'. Eminem is Marshall Mathers, but since this cannot be known a priori, 'Eminem is not taller than Marshall Mathers' does not possess the Kripkean property. Our knowledge that it is necessary stems from our empirical knowledge that Eminem is Marshall Mathers.

If we go along with what these authors say about their examples - and I see no strong objections - we must admit that they lack the Kripkean property. But they do not falsify (Link), since they are both implied by true propositions with the Kripean property. We get such implying propositions by simply conjoining the examples with a statement of the empirical knowledge which enables us to know their modal status. So, 'Air is airy stuff and all there is to being air is being airy stuff' is true, it possesses the Kripkean property, and it implies Kipper's example. Similarly, 'Eminem is not taller than Marshall Mathers and Eminem is Marshall Mathers' is true, it possesses the Kripkean property, and it implies Strohminger \& Yli-Vakkuri's example.

Why do these conjunctions possess the Kripkean property? Take the Eminem case. Regarding the conjunction 'Eminem is not taller than Marshall Mathers and Eminem is Marshall Mathers', we can do the following a priori reasoning. Suppose this is true. Then both conjuncts are true. Since the second conjunct is true, the first conjunct is necessarily true. And we know by Kripke's familiar analysis that the second conjunct is necessarily true if true at all, so it is necessarily true also, making the whole conjunction necessarily true. Thus the conjunction is necessarily true if true at all. ${ }^{7}$

\footnotetext{
${ }^{7}$ Thanks to Albert Casullo and Jens Kipper for encouraging discussion, and to two anonymous referees for this journal who uncovered an error in the submitted version of this paper.
} 


\section{Conflict of interest statement}

On behalf of all authors, the corresponding author states that there is no conflict of interest.

\section{References}

Anderson, C. Anthony (1993). Toward a Logic of A Priori Knowledge. Philosophical Topics 21(2):1-20.

Casullo, Albert (2003). A Priori Justification. Oxford University Press USA.

Casullo, A. (forthcoming). Modal empiricism: What is the problem?. Oxford Studies in Epistemology, Vol. 6.

Chalmers, David J. (2006). Two-dimensional semantics. In E. Lepore \& B. Smith (eds.), Oxford Handbook of the Philosophy of Language. Oxford University Press.

Chalmers, David J. (2006b). The Foundations of Two-Dimensional Semantics. In Manuel Garcia-Carpintero \& Josep Macia (eds.), Two-Dimensional Semantics: Foundations and Applications. Oxford University Press. pp. 55-140.

Chalmers, David J. (2011). The nature of epistemic space. In Andy Egan \& Brian Weatherson (eds.), Epistemic Modality. Oxford University Press.

Kipper, Jens (2018). On what is a priori about necessities. Analysis 78 (2):235-243.

Kripke, Saul A. (1971). Identity and necessity. In Milton K. Munitz (ed.), Identity and Individuation. New York University Press. pp. 135-164.

Kripke, Saul A. (1980). Naming and Necessity. Harvard University Press.

Peacocke, Christopher (1998). Being Known. Oxford University Press.

Strohminger \& Yli-Vakkuri (m.s.). On A Priori Knowledge of Necessity. $<$ https://philpapers.org/rec/YLIOAP> (Last accessed 18 March 2018.) 